\title{
Neurotologic and Functional MRI Findings in a Patient With Bilateral Profound Deafness Having Brown-Vialetto-Van Leare Syndrome
}

\author{
*Cinzia Salmina, $†$ Franca Wagner, $†$ Roland Wiest, $\ddagger$ Andrea Federspiel, \\ *Pascal Senn, *Marco Caversaccio, and *Dominique Vibert \\ *Department of Otorhinolaryngology, Head and Neck Surgery; $\dagger$ Department of Diagnostic and Interventional \\ Neuroradiology; and $\ddagger$ Department of Psychiatric Neurophysiology, University Hospital of Psychiatry, \\ Inselspital Bern, Switzerland
}

\begin{abstract}
Introduction: We report the first findings of functional magnetic resonance imaging of the auditory cortex in a young woman with a bilateral cochleovestibular deficit as first manifestation of Brown-Vialetto-Van Leare syndrome. The patient had no open speech discrimination, even with hearing aids, and is depending on lip reading for communication.

Methods: To evaluate the possible efficiency of a cochlear implantation, we investigated hemodynamic responses within the central auditory pathways using an auditory functional magnetic resonance imaging paradigm.
\end{abstract}

Results: Blood oxygen level-dependent correlates were detected bilaterally along the auditory pathways after exposure to intermittent clicking tone stimulation at $2 \mathrm{kHz}$.

Conclusion: These results suggest integrity of the central auditory pathways and represent a positive argument to propose a cochlear implantation with the aim to restore hearing. Key Words: Brown-Vialetto-Van Leare syndrome-Functional magnetic resonance imaging.

Otol Neurotol 35:1495-1500, 2014.
The Brown-Vialetto-Van Leare syndrome (BVVL) is a very rare disorder characterized by bilateral progressive sensorineural deafness and deficits involving the motor components of the lower cranial nerves. Since the first clinical description by Charles Brown in 1894 (1), only 58 cases have been reported in literature.

The etiology of the disease is unknown, but familial cases suggest an autosomal recessive inheritance (2). The diagnosis of BVVL is very difficult because of its rarity and the lack of confirmatory tests. Until now, there is no specific treatment regimen evaluated and the patients are treated symptomatically.

Using functional magnetic resonance imaging (fMRI), we reported the first case of BVVL with a positive response of central auditory pathways on both sides.

\section{CLINICAL PRESENTATION}

In January 2013, a 30-year-old woman with BVVL was examined at our tertiary ENT referral center with a history of bilateral progressive hearing loss and balance disorder.

\footnotetext{
Address correspondence and reprint requests to Franca Wagner, University Hospital, Bern, Switzerland; E-mail: franca.wagner@insel.ch Cinzia Salmina and Franca Wagner shared first authorship.

The authors disclose no conflicts of interest.
}

The patient reported a progressive bilateral hearing loss since 2002. She complained of severe hearing loss and, despite her hearing aids, she was dependent on lip reading to communicate. Moreover, for 1 year, she suffered from recurrent vertigo attacks with dizziness, especially during movement as well as by walking in darkness. BVVL was first diagnosed in 2011. She was treated with an experimental therapy of high doses of steroids and immunoglobulins but without improvement of her symptomatology.

On examination, the patient was oriented and conscious with no mental deterioration. The neurologic examination showed tongue atrophy and fasciculation leading to slurring of speech as a sign of the bulbar palsy. The other cranial nerves were normal on clinical testing. The cerebellar testing showed hypometria in the finger-to-nose test. In addition, the patient presented spastic quadriplegia, with legs being tighter than arms, tetrahyperreflexia, and a positive Babinski sign. In the Romberg's test, the patient showed a strong tendency to fall to the left side; the Unterberger's test was not performed because of the strong instability. The clinical vestibular examination showed neither spontaneous nor positional nystagmus.

A complete neurotologic examination was performed, including pure-tone audiogram, brainstem evoked auditory potentials (BEAPs), videonystagmography, and cervical vestibular-evoked myogenic potentials. The 


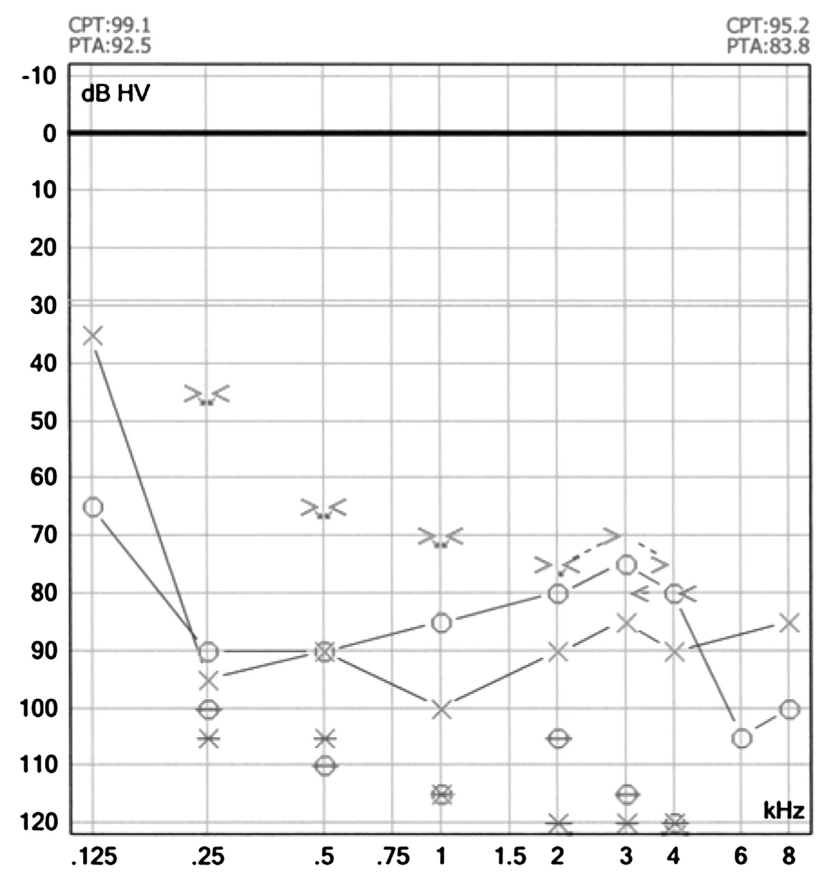

FIG. 1. The pure-tone audiogram shows a profound bilateral sensorineural deafness.

pure-tone audiogram confirmed the bilateral profound sensorineural hearing loss (Fig. 1). The BEAP performed with liminal thresholds of 95 dBSPL showed desynchronized curves on both sides (Fig. 2). The videonystagmography recorded a transient rotatory nystagmus during the Rose maneuver on the right side and a transient up-beating nystagmus during the Rose maneuver on the left side. Bithermal caloric test showed a left hyporeflexia and a right areflexia.
Measurement of cervical vestibular-evoked myogenic potentials revealed no response on both sides.

Taken together, the neurotologic findings showed a bilateral sensorineural hearing loss retrocochlear in origin as well as a bilateral peripheral vestibular deficit involving both canal and otolithic functions.

To exclude a structural origin of the progressive bilateral hearing loss in our patient with known BVVL syndrome, a
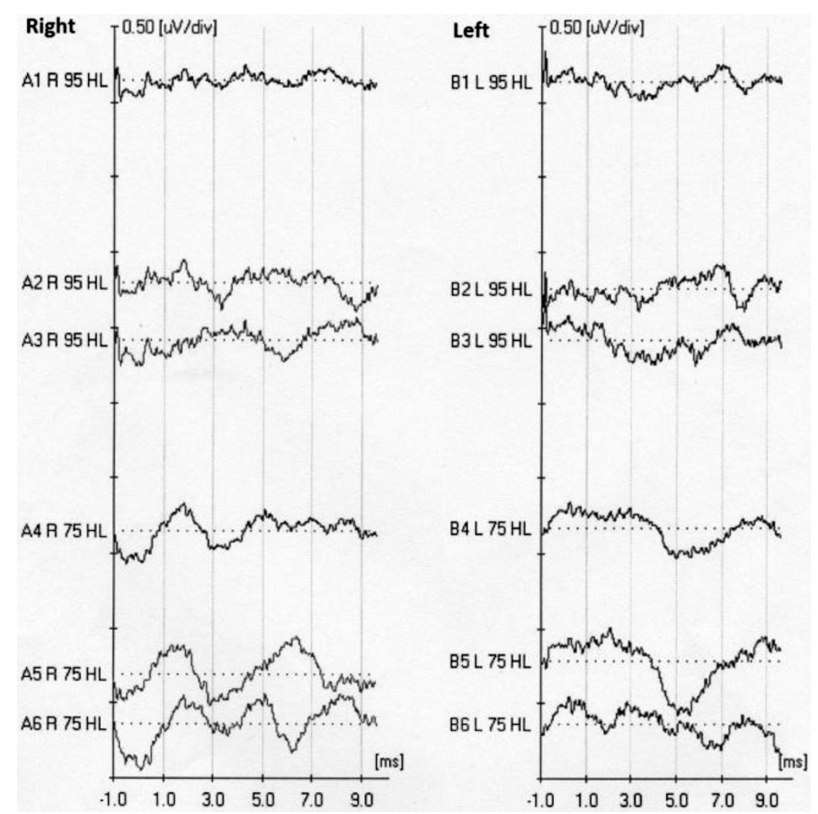

FIG. 2. Brainstem evoked auditory potential findings with liminar stimulation next to hearing threshold of $95 \mathrm{dBSPL}$ (sound pressure level) showed desynchronization of curves on both sides. 
baseline MRI of the head with complementary highresolution three-dimensional constructive interference in steady state sequence (3D-CISS; $0.5-\mathrm{mm}$ slice thickness) of the skull base was previously performed in June 2006 to evaluate the cranial nerve VIII and its branches along the cerebellopontine angle and internal auditory canal bilaterally (Fig. 3). The signal intensity of the fluid in the membranous labyrinth revealed no signal changes on T2weighted constructive interference in steady state sequences. That initial MRI showed bilaterally a normal configuration and width of the internal acoustic meatus in anteroposterior (AP) measurement (maximum AP right, $5.3 \mathrm{~mm}$; maximum AP left, $5.5 \mathrm{~mm}$ ) in keeping with normative values (3). The four branches of the vestibulocochlear nerve in the internal auditory canal were identified bilaterally; particularly, the cochlear nerve on both sides appeared normal with a maximum diameter of $1.1 \mathrm{~mm}$ on the right and on the left of $1.0 \mathrm{~mm}$ (4). The root-entry zone of the vestibulocochlear nerve was inconspicuous on both sides. After intravenous contrast application, there were no abnormalities MR morphologically. The signal intensity of the membranous labyrinth was regular, and no evidence of labyrinthic malformations were found on both sides. Because of the rapidly progressive hearing loss, the patient received several follow-up MRI examinations, focusing on the temporal skull and structures, respectively, along the cerebellopontine angle. The last MRI examination of the skull base for that patient was performed in January 2013 during the fMRI examination. Overall, in these 79month follow-up, the cochlear nerve on both sides continued to provide unenhanced and enhanced examinations inconspicuous. There were also no changes of the normal hyperintense fluid signal in the membranous labyrinth bilaterally.

The fMRI with an auditory stimulation protocol with intermittent presentation of auditory stimuli using a monofrequent click tone at $2 \mathrm{kHz}$ frequency (the control condition was simply "no sound") repeated twice a second was performed as a complementary diagnostic

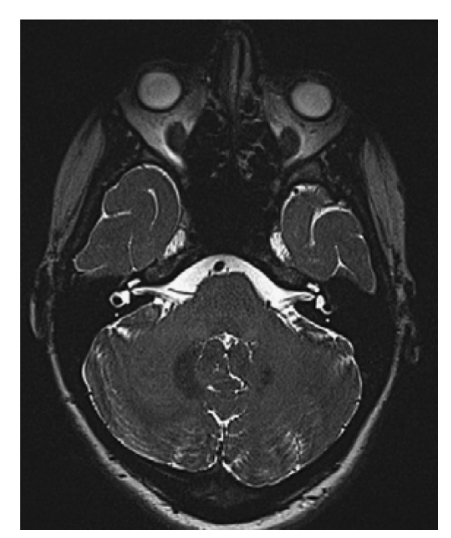

FIG. 3. The three-dimensional constructive interference in steady state (3D-CISS) showed a normal appearance of the vestibulocochlear nerve in the cisternal segment and inside the internal auditory canal bilaterally. imaging to accurately verify hemodynamic responses within the auditory pathways. The presentation at the chosen frequency relied on a previous evaluation where we tested click-tone stimuli alternating between the right ear versus the left ear. The rationale to use monofrequent tones at the given frequency was to produce a consistent stimulus at a maximum suprathreshold level that could be provided by the sound system of "MR confon GmbH" (Magdeburg, Germany; http://www.mr-confon.de/en/products.html). The earphones used in the present study are exactly the same as described in Baumgart et al. (5). In their analysis, these earphones were extensively tested and measured concerning sound pressure level (SPL) (5). The earphones used in the present study are suited for noise protection because they are built with an active noise-suppression design without hindering the efficient transmission of sound (5). Ear muffs with liquid-filled rims suppress the gradient-induced noise from fMRI sequences. This system ensures adequate noise suppression without the need for earplugs that are normally applied in conventional fMRI investigations. The active noise-suppression mechanism of the earphones used is so efficient that the fMRI noise cannot be substantially detected and perceived.

We used a standard block design with five repetitive measures for every condition to improve the signal-to-noise ratio compared with an event-related design. The interleaved acquisition of the blood oxygen level-dependent (BOLD) sequence parameters in the block design is robust and does not negatively affect the signal-to-noise ratio. To prove this, the calculation of percentage of signal changes is demonstrated (Fig. 4). To prove the integrity of the auditory pathways, we added the HR along the crucial subcortical nuclei of the auditory pathway in response to the stimuli. Confounders caused by low-frequency oscillations caused by rs activity in the range of 0.01 to $0.1 \mathrm{~Hz}$ can be excluded because we applied high-pass filtering ahead of analysis.

In addition, the fMRI was performed on a $3 \mathrm{~T}$ Siemens Magnetom Trio TIM system (Siemens AG, Erlangen, Germany). High-resolution three-dimensional volume images were obtained using a multiplanar rapid gradient echo sequence (MP-RAGE; 176 sagittal slices; isovoxel resolution, $1.0 \mathrm{~mm}$; FOV, $256 \times 256 \mathrm{~mm}$; matrix size, $256 \times$ 256; TR/TE/TI, 1, 950/2.15/900 ms). The fMRI was performed with a multislice single-shot $\mathrm{T} 2 *$-weighted echo planar imaging sequence ( 35 slices; slice thickness, $4 \mathrm{~mm}$; gap thickness, $0.75 \mathrm{~mm}$; TR/TE, $6,000 / 50 \mathrm{~ms}$; matrix size, $128 \times 128 \mathrm{~mm}$; FOV, $192 \times 192 \mathrm{~mm}$; flip angle, 90 degrees), with a total of 60 volumes. The fMRI BOLD contrast measurements were performed in two runs, separately for the left and right ear, with cycles of 30 seconds "tone" (up to a maximum SPL of $82 \mathrm{~dB}$ ) and 30 seconds "silent" repeated seven times (four cycles "tone" and three cycles "silent"), resulting in a total presentation time of 420 seconds. The computer automatically triggered alternation. Voxel-wise correlations between the BOLD signal and the predictor were computed with a general linear model. The six motion parameters derived from the fMRI preprocessing (translation and rotation in the $x, y$, and $z$ directions) were used as covariates in the general 


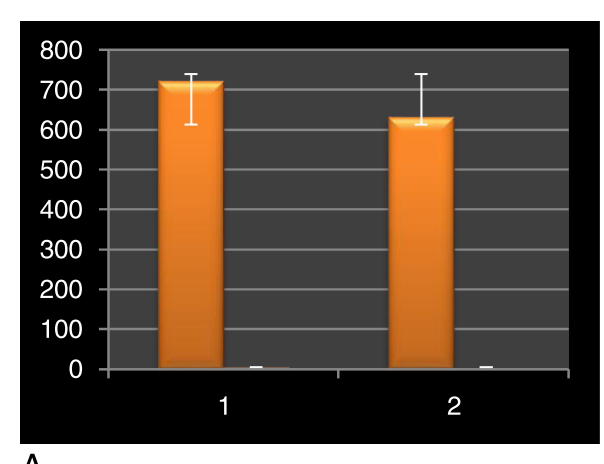

A

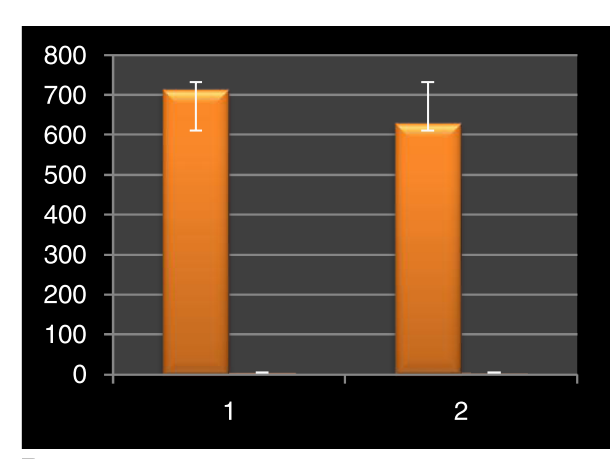

B

FIG. 4. The calculation of percentage of signal changes for the left side $(A)$ and the right side $(B)$.

linear model. Correction for multiple comparisons and false-positives was performed with a false discovery rate threshold of $p<0.05$.

Bilateral hemodynamic correlates were detected in the transverse temporal gyrus with left-sided stimulation correlation to a greater extent with the stimulation paradigm than right-sided stimulation (Fig. 5). Overall, fMRI confirmed the integrity of the central auditory pathways of both hemispheres.

\section{DISCUSSION}

Progressive hearing loss as first symptom of BVVL usually begins within the third decade (6). Our patient was 20 years old when the bilateral hearing loss occurred. Ten years later, the cochlear function was so deteriorated that she was dependent on lip reading for speech comprehension. The pure-tone audiometry confirmed the bilateral profound sensorineural hearing loss. Pathologic findings of BEAP were interpreted as the electrophysiologic sign of the bilateral auditory neuropathy.

Vestibular symptoms caused by the bilateral vestibular neuropathy occurred about 10 years after the onset of the hearing loss. The late occurrence of the vestibular symptomatology was interpreted as caused by the fact that the brain was first able to compensate for the progressive alteration of the vestibular function.

Because of the very poor speech discrimination, the cochlear implantation (CI) procedure was discussed. Indeed, CIs have been reported to be a valid therapeutic option with retrocochlear severe hearing loss such as in case of superficial hemosiderosis (7) and auditory neuropathy spectrum disorder $(8,9)$. However, deaf patients with an abnormal auditory nerve function need to be counseled that postoperative results are more variable and less predictable compared with implanted patients with cochlear deafness.

The fact that fMRI in this special case revealed the integrity of the central auditory pathways is one positive argument in favor for CI. This argument is really needed because in the literature, to our knowledge, only one article reported CI in two patients with BVVL, but both without postoperative improvement of hearing (10). In both cases, the central auditory pathways were not evaluated by fMRI before implantation.

Of course we may infer from the behavioral response that our patient is hearing. The same holds true for fMRI studies in brain tumors and epilepsy, where we know that the patients are aphasic or generate seizures. The incentive to perform the fMRI was to provide converging evidence on the basis of the integration of functional information that resembles the knowledge about altered physiology of cortical and subcortical transmission in the auditory pathways. Functional MRI plays already an essential role for quality assurance of the treatment if the subjective response of a patient may be ambiguous. The same features are already routinely used in the field of neurology and psychiatry, for example, in patients with psychogenic blindness or in functional hemiparesis $(11,12)$. Beyond behavioral responses, fMRI provides additional information on the integrity of auditory pathways along the brainstem and thalamic pathways in keeping with the structural information from highresolution MRI. To clarify this, we have added two images that indicate the responses to the auditory stimulation along the right and left auditory pathways (Fig. 5). Furthermore, it should be mentioned that, by using fMRI responses, there is an operator-independent way of quantifying the presence or absence of an auditory response. In these cases, neurotologists not only know that, yes, behavioral responses in both ears already show that the auditory nerve and auditory cortex are capable of transmitting some degree of sound. Neurotologists here additionally may quantify the amount of "neuronal" response to stimuli. This is clearly not only a yes/no discrimination but an additional insight into the neuronal functioning in the proximity of the relevant brain region (although fMRI is not a direct measure of neuronal activity). Moreover, fMRI can be a valuable tool when patients need to be prepared for an intervention such as in our case a cochlear implant in which the actual activation pattern to a stimulus may be visualized like in our case for decision making for or against the cochlea implant and which side should be preferred for the CI. Our candidate is aware of the implanted cases described in the literature and seeks more information before deciding whether she wants to undergo an implantation or not. Some limiting factors may be considered when interpreting the MRI findings. The study 

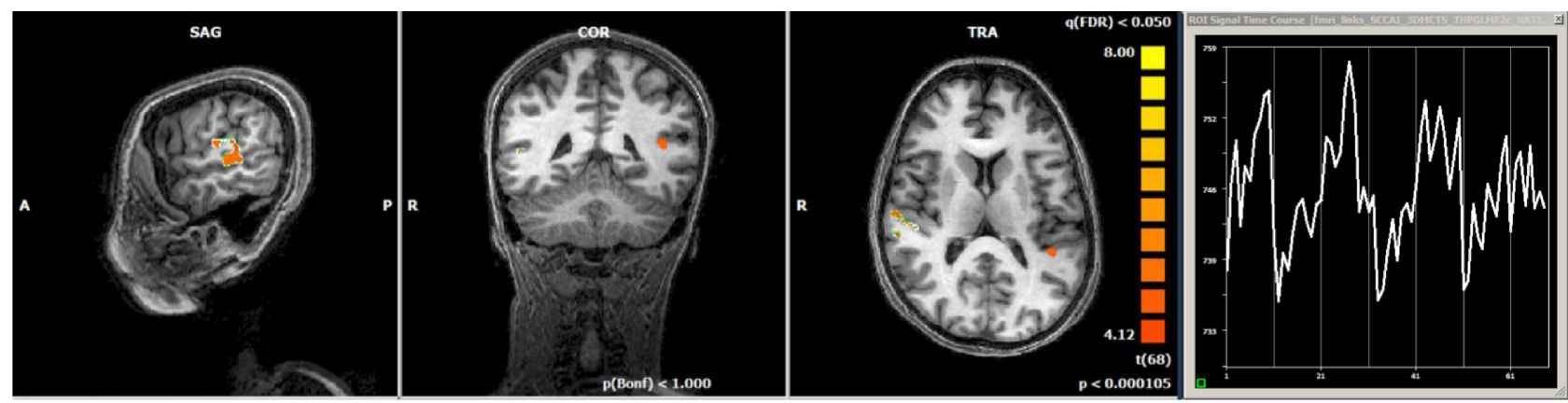

A
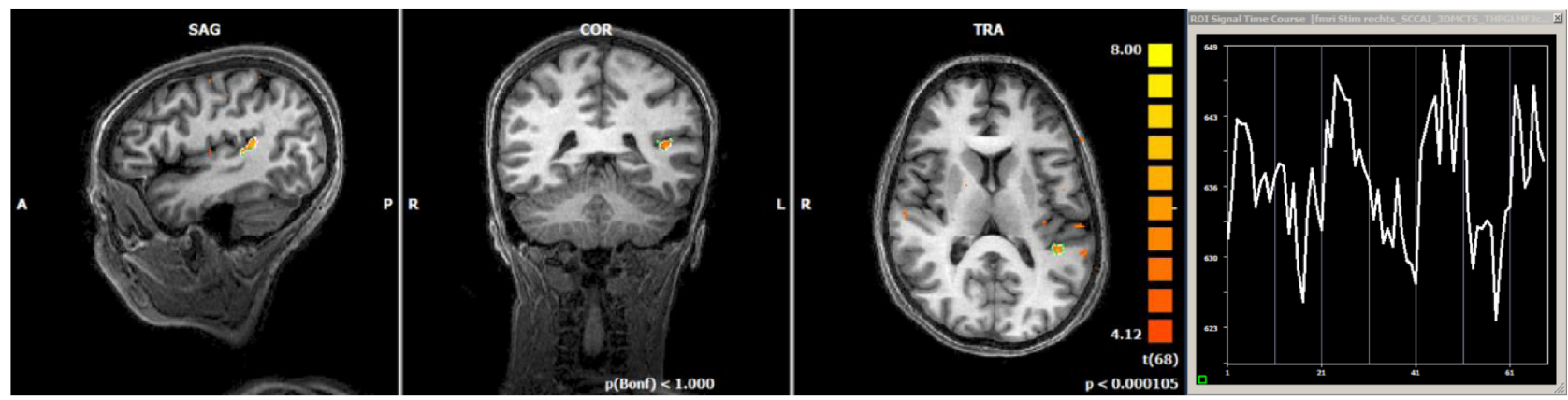

B
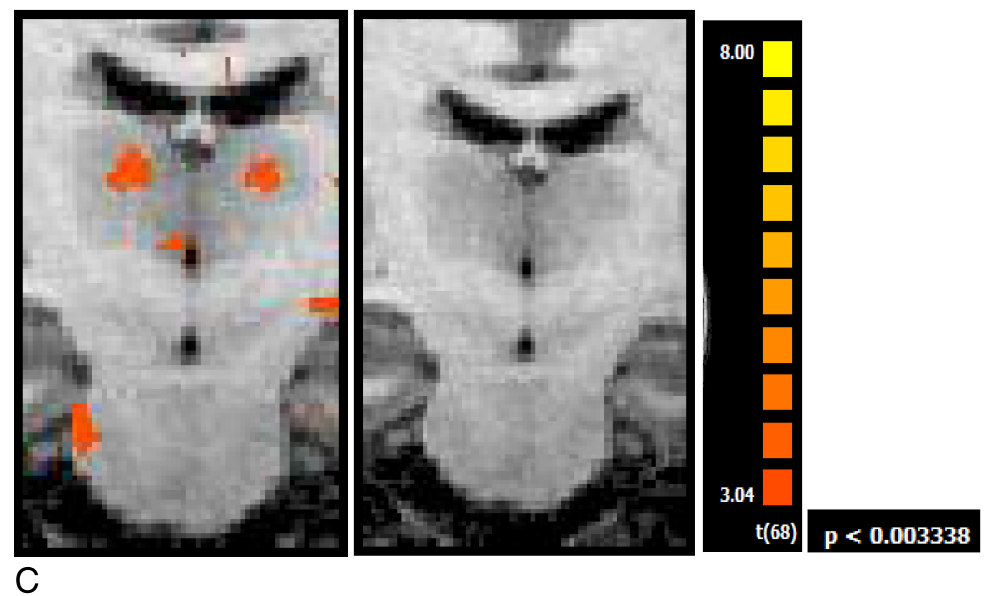

FIG. 5. Functional magnetic resonance imaging demonstrates bilaterally hemodynamic correlates in the transverse temporal gyrus after selective acoustical stimulation by clicking sound presentation $(2 \mathrm{kHz})$ with left-sided $(A)$ correlation to a greater extent with the stimulation paradigm than right-sided $(B)$. $C$, The responses to the auditory stimulation along the right and left auditory pathways.

was performed within an fMRI environment with known noise conditions. Therefore, the stimulus delivery may not be compatible with "audiologic standards" that would be required in a setting for hearing assessment. We are aware that this poses limitations to the stimulus delivery. In fact, a calibration of the earphones by measuring SPL may not be performed like in standard laboratory conditions (the MR-compatible optical microphone is one of the limiting factors; the exact sound pressure on the measurement surface may be contaminated by the surrounding sequence noise; standard temperature and standard environmental pressure may not be maintained constant). Beside these shortcomings, the fMRI signal clearly captures hemodynamic response to a stimulus as compared with resting periods. We assume that, by computing the percentage of signal changes between these two conditions, the effect of the systematic errors cancels out because both conditions are equally affected by these systematic errors.

\section{CONCLUSION}

Bilateral progressive cochleovestibular deficit caused by BVVL is rare but must be suspected in case of failure of other lower cranial nerve functions. Functional MRI investigating hemodynamic responses to auditory stimulation in the transverse temporal gyrus is an alternative approach to 
neurophysiologic techniques to investigate central auditory pathways in case of auditory neuropathy. Positive imaging represents an objective argument to propose and discuss the CI procedure as rehabilitation of hearing. Nevertheless, more findings are needed to confirm this first case report.

\section{REFERENCES}

1. Brown $\mathrm{CH}$. Infantile amyotrophic lateral sclerosis of the family type. J Nerv Ment Dis 1894;21:707-16.

2. Megarbane A, Desguerres I, Rizkallah E, et al. Brown-Vialetto-Van Laere syndrome in a large inbred Lebanese family: confirmation of autosomal recessive inheritance? Am J Med Genet 2000;92:117-21.

3. Erkoç MF, Imamoglu H, Okur A, Gümüș C, Dogan M. Normative size evaluation of internal auditory canal with magnetic resonance imaging: review of 3786 patients. Folia Morphol (Warsz) 2012;71:217-20.

4. Nakamichi R, Yamazaki M, Ikeda M, et al. Establishing normal diameter range of the cochlear and facial nerves with 3D-CISS at $3 \mathrm{~T}$. Magn Reson Med Sci 2013;0004:1-7.

5. Baumgart F, Kaulisch T, Tempelmann C, et al. Electrodynamic headphones and woofers for application in magnetic resonance imaging scanners. Med Phys 1998;25:2068-70.
6. Francis DA, Ponsford JR, Wiles CM, Thomas PK, Duchen LW. Brown-Vialetto-Van Laere syndrome. Neuropathol Appl. Neurobiol 1993;19:91-4.

7. Berrettini S, De Vito A, Bruschini L, Fortunato S, Forli F. Cochlear implantation in patients affected by superficial hemosiderosis of the central nervous system. Eur Arch Otorhinolaryngol 2012;269:25-31.

8. Jeon JH, Bae MR, Song MH, Noh SH, Choi KH, Choi JY. Relationship between electrically evoked auditory brainstem response and auditory performance after cochlear implant in patients with auditory neuropathy spectrum disorder. Otol Neurotol 2013;34:1261-6.

9. Humphriss R, Hall A, Maddocks J, Macleod J, Sawaya K, Midgley E. Does cochlear implantation improve speech recognition in children with auditory neuropathy spectrum disorder? A systematic review. Int J Audiol 2013;52:442-54.

10. Sinnthuray AR, Watson DR, Fruhstorfer B, Oloarte JR, Toner JG. Cochlear implantation in Brown-Vialetto-Van-Laere syndrome. J Laryngol Otol 2011;125:314-7.

11. Vuilleumier P. Hysterical conversion and brain function [Review]. Prog Brain Res 2005;150:309-29.

12. Werring DJ, Weston L, Bullmore ET, Plant GT, Ron MA. Functional magnetic resonance imaging of the cerebral response to visual stimulation in medically unexplained visual loss. Psychol Med 2004;34:583-9. 